\title{
Meaning Banking beyond Events and Roles Invited talk
}

\author{
Johan Bos \\ University of Groningen \\ johan.boserug.nl
}

\begin{abstract}
In this talk I will discuss the analysis of several semantic phenomena that need meaning representations that can describe attributes of propositional contexts. I will do this in a version of Discourse Representation Theory, using a universal semantic tagset developed as part of a project that aims to produce a large meaning bank (a semantically-annotated corpus) for four languages (English, Dutch, German and Italian).
\end{abstract}

